\title{
O uso do e-mail na comunicação com o médico de família: catorze meses de experiência
}

Mónica Granja*

\section{RESUMO}

Objectivos: Caracterizar as mensagens de correio electrónico entre uma médica de família (MF) e os seus pacientes durante os primeiros 14 meses de acesso.

Estudo: Transversal descritivo

Local: Centro de Saúde da Senhora da Hora

População: Mensagens de e-mail trocadas entre os pacientes e a sua MF.

Métodos: Uma amostra aleatória de $20 \%$ das mensagens recebidas foi estudada quanto a: sujeito da mensagem, dimensão, número e tipo de assuntos abordados, existência de resposta do MF e dimensão desta, intervalo de tempo até à resposta, hora de envio e resultado das mensagens. Fez-se a caracterização sócio-demográfica e familiar de todos os utilizadores. Determinou-se o tempo gasto com o e-mail numa semana. Na análise estatística descritiva usou-se o programa Excel 2003.

Resultados: Foram recebidas 504 mensagens, de 92 utilizadores diferentes, com idade média de 37,1 anos, sendo 73\% do sexo feminino e $65 \%$ com escolaridade superior. Foram enviadas pela MF 462 mensagens. As 100 mensagens estudadas visavam 112 sujeitos (mais frequentemente o próprio, filhos menores, idosos dependentes ou cônjuge). As mensagens tinham em média 57,1 palavras e abordavam 1,3 assuntos (mais frequentemente questões médicas, de exames complementares, administrativas e de enfermagem e de medicação). A MF respondeu a $69 \%$ das mensagens e em $94,2 \%$ dos casos fê-lo em menos de 48 horas, usando em média 58,2 palavras. Das mensagens recebidas, $22 \%$ foram escritas fora do horário do CS. A MF enviou $58 \%$ das suas mensagens em horas não assistenciais. Os resultados mais frequentes da troca de mensagens foram: resposta com informação médica ou aconselhamento, emissão de receitas, credenciais para exames ou tratamentos e atestados, marcação de consulta e referenciação. Numa semana, gastou-se uma média diária de 5 minutos e 31 segundos com o e-mail.

Conclusão: Os resultados deste estudo coincidem com os referidos noutros países: pacientes e cuidadores, sobretudo mulheres, activas e instruídas, aderem ao e-mail para comunicar com o seu MF, sem trazerem sobrecarga de trabalho. Estes resultados poderão favorecer a adesão de mais MF ao e-mail e o investimento dos CS nesta ferramenta.

Palavras-chave: E-mail; Comunicação; Acessibilidade.

\section{INTRODUÇÃO}

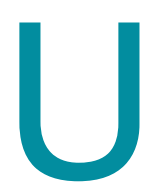

ma das componentes da acessibilidade (característica fundamental da disciplina da Medicina Geral e Familiar) ${ }^{1,2}$ é a possibilidade de contacto não presencial com a equipa de saúde. ${ }^{3-5}$ Tradicionalmente, o contacto não presencial com o médico de família (MF) é feito via telefo-

*Assistente Graduada de Medicina Geral e Familiar

Centro de Saúde da Senhora da Hora

Unidade Local de Saúde de Matosinhos ne mas a insatisfação dos utentes com a acessibilidade telefónica ao Centro de Saúde (CS) e ao MF parece ser frequente. ${ }^{6,7}$ Num estudo num CS português as percentagens de satisfação na dimensão acessibilidade telefónica rondaram os $30 \%$ (para uma média de satisfação de $70 \%$ nas restantes dimensões). ${ }^{7}$ A interrupção das consultas pelo telefone conduz à frequente limitação das horas disponíveis para contacto telefónico, reduzindo muito o potencial de acessibilidade deste meio de comunicação. 
Um muito citado relatório do Institute of Medicine (órgão da Academia de Ciências norte-americana, entidade não governamental e sem fins lucrativos) acerca da evolução em qualidade do sistema de saúde norte-americano recomendava explicitamente que os pacientes tivessem acesso electrónico a profissionais de saúde. ${ }^{8}$ Estudos têm revelado que o correio electrónico é preferido ao telefone, quer por médicos, quer por pacientes, perante problemas não urgentes, gerando satisfação entre pacientes, cuidadores e médicos, ${ }^{9-15}$ sendo referido como facilitador, ou menos intimidador, para a abordagem de questões mais sensíveis ${ }^{12}$ ou para o fornecimento de informação por parte de cuidadores, ${ }^{16}$ sem induzir utilização excessiva e sem aumentar o tempo dispendido pelo médico, ${ }^{9,10,13,17,18}$ e melhorando os cuidados a doentes crónicos, a continuidade e a flexibilidade.${ }^{16}$ Num inquérito em 2002, a grande maioria dos norte-americanos referiu que gostaria de ter acesso ao seu médico por $e$-mail e, para mais de metade dos inquiridos, isso interferiria na opção por determinado médico. ${ }^{19}$ Autores norte-americanos relataram que um paciente se referiu às mensagens de $e$-mail como sendo «a melhor coisa que há, logo a seguir a uma consulta ao domicílio» ${ }^{20}$ e que os pais de uma criança comentaram «o e-mail traz o médico a nossa casa». ${ }^{18}$

$\mathrm{O}$ uso do correio electrónico apresenta a vantagem ímpar de constituir um registo clínico em si mesmo, possibilitando que traga anexos de fotografias (por exemplo de lesões cutâneas) ou de vídeos (por exemplo de uma tosse), existindo plataformas que o incorporam automaticamente nos demais registos electrónicos do paciente. Em sentido inverso, podem ser enviados pelo médico folhetos de educação para a saúde e guias para pacientes, assim como avisos gerais (por exemplo, de férias ou alterações organizacionais) ou mesmo pedidos de colaboração.

Segundo dados da agência Eurostat, ${ }^{21} 46 \%$ das famílias portugueses tinham acesso à internet $\mathrm{em} 2008$. Embora situando-se este valor abaixo dos $60 \%$ da média europeia, ele corresponde a um crescimento acelerado, triplicando os 15\% de 2002. Para quem tem acesso à internet, o correio electrónico é gratuito, fácil de usar e conveniente para emissor e receptor ao possibilitar contactos rápidos mas diferidos no tempo segundo a conveniência de cada um.

Apesar das vantagens enunciadas, é do conhecimen- to geral que muitos CS ainda não disponibilizam contas de $e$-mail aos seus profissionais e que a maioria dos MF não usa esta ferramenta sequer a título pessoal. Além disso, a prática do uso do correio electrónico entre médicos e pacientes é questionada, mesmo por médicos de família habituados ao manejo das tecnologias de informação, como foi patente em discussões como as que ocorreram online no $" 1^{\circ}$ Congresso Virtual de Medicina Geral e Familiar» (organizado em Portugal em 2008 e que já não está em linha) ou como as que vêm, ciclicamente, a lume, no fórum electrónico MGFamiliar (dado ser um fórum de acesso restrito a MF registados não é possível incluir as referências).

Os obstáculos levantados pelos que não são adeptos do uso desta ferramenta prendem-se com: trabalho não contabilizado nem remunerado; medo de sobre-utilização pelos pacientes e de sobrecarga de trabalho; falta de à vontade com nova tecnologia; impressão de despersonalização da relação médico-paciente; medo de que a linguagem escrita não sirva a comunicação desejável; questões de confidencialidade; e falhas frequentes nos sistemas de informação. ${ }^{14-16,22,23}$

Numa pesquisa na PubMed e no Índice de Revistas Médicas Portuguesas, não foi encontrado nenhum artigo português sobre uso clínico do correio electrónico. A autora, MF, decidiu, assim, realizar e divulgar um estudo sobre a sua experiência do uso de correio electrónico com os seus pacientes, com vista ao melhor conhecimento desta prática e a proporcionar um debate sobre pontos positivos e negativos, oportunidades de melhoria e eventuais ameaças a ter em conta.

O acesso ao correio electrónico da MF autora do estudo está disponível para os pacientes da sua lista desde Outubro de 2006. A implementação do serviço começou com a divulgação deste acesso feita através de aviso (incluindo algumas orientações para a sua utilização), o qual foi afixado na sala de espera, disponibilizado no balcão administrativo e oferecido de forma oportunista durante as consultas. Nos primeiros meses foi utilizada uma conta pessoal da autora, especialmente aberta para o efeito e à qual acedia a partir de casa. A partir de Abril de 2007, passou a dispor de uma conta de correio electrónico no seu posto de trabalho (consultório do CS partilhado com outra colega), sendo a alteração comunicada nos meios de divulgação atrás descritos e ainda pelo envio do novo endereço a todos os 
pacientes que já tivessem usado o sistema.

\section{MÉTODOS}

Com o objectivo de caracterizar as mensagens de correio electrónico trocadas entre uma MF e os pacientes da sua lista ao longo de 14 meses de acesso ao sistema, realizou-se um estudo transversal descritivo sobre as mensagens de correio electrónico trocadas entre 20/04/2007 e 20/6/2008, conduzido pela própria MF. Foram contabilizados os dias de trabalho efectivo no período do estudo, recolhidos os números totais de mensagens recebidas de pacientes e enviadas pela MF e feita a caracterização sócio-demográfica e familiar dos seus pacientes utilizadores. Utilizando o programa informático Research Randomizer, foi recolhida uma amostra aleatória de $20 \%$ das mensagens recebidas de pacientes, as quais foram estudadas quanto a sujeito(s) acerca de quem a mensagem era escrita (próprio ou outros), dimensão (em número de palavras), número e tipo de assuntos abordados, segundo uma adaptação de taxonomias utilizadas em estudos semelhantes, ${ }^{15,24}$ existência de mensagem de resposta da MF e respectiva dimensão (em número de palavras), intervalo de tempo entre envio de mensagem pelo utilizador e envio da resposta pela MF (excluindo sábados, domingos e feriados), hora de envio das mensagens recebidas e das respectivas respostas, resultado das mensagens trocadas (ausência de qualquer acção, emissão de recei- tas, credenciais para MCD ou tratamento ou atestados, informações clínicas e aconselhamento, marcação de consulta ou outros).

Foi ainda determinado, por cronometragem, na última semana do estudo, o tempo gasto pela MF a ler e a responder a mensagens.

Foi feita análise estatística descritiva usando o programa Excel 2003, determinando-se a média, mediana, desvio-padrão e intervalo para as variáveis contínuas e as percentagens para as variáveis categóricas.

Para uma melhor contextualização dos resultados, foram ainda recolhidos alguns dados da prática da MF autora.

\section{RESULTADOS}

\section{Descrição do movimento de mensagens}

Nos primeiros 14 meses (de 20/04/2007 a 20/06/2008) de acesso ao correio electrónico através da conta do CS, foram recebidas 504 mensagens de pacientes (quatro mensagens de «lixo electrónico» não foram contabilizadas). O período indicado correspondeu a 242 dias efectivos de trabalho, o que resulta numa média de 2,08 mensagens por dia. Esta média encerra, contudo, uma tendência crescente do número de mensagens por dia e por mês ao longo do período do estudo, sendo inferior a uma mensagem por dia nos primeiros dois meses (completos) estudados e superior a três mensagens por dia nos últimos quatro meses (Figura 1).

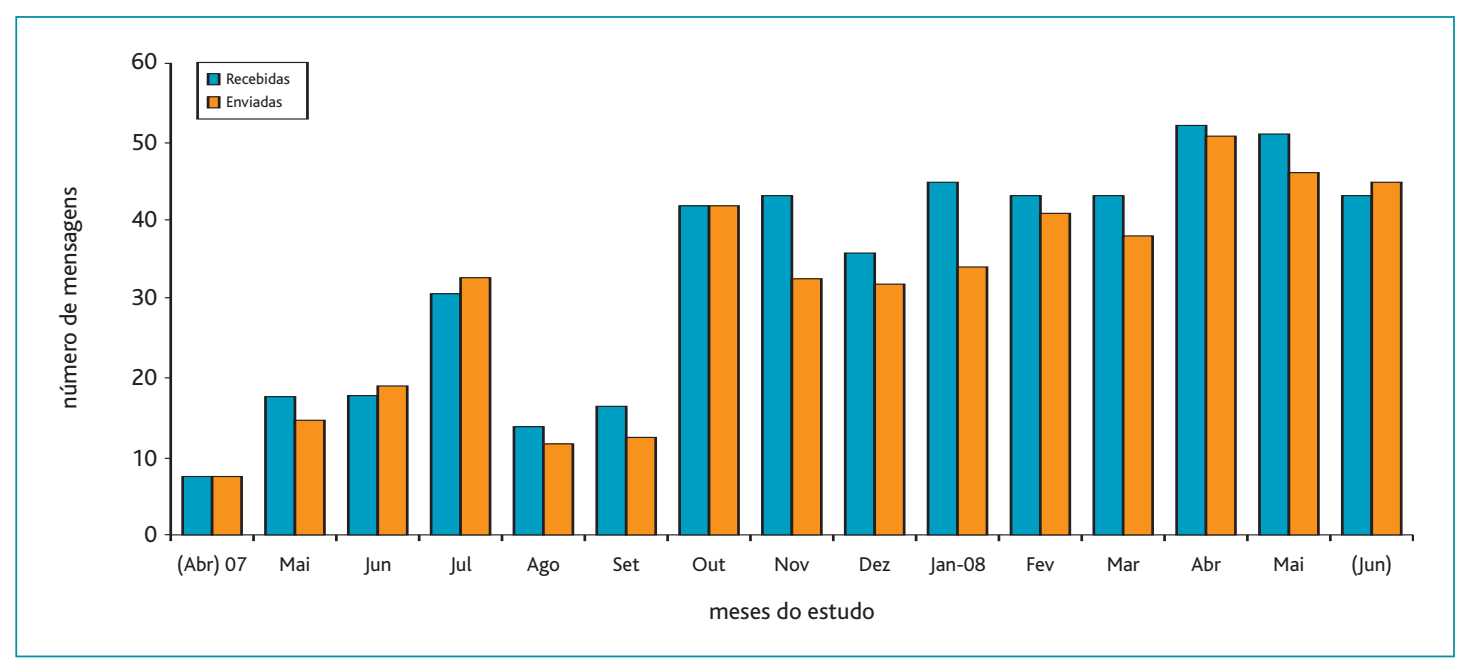

Figura 1. Evolução do número mensal de mensagens recebidas e enviadas ao longo dos meses estudados. 


\begin{tabular}{|c|c|c|}
\hline \multicolumn{3}{|c|}{$\begin{array}{l}\text { QUADRO I. Características sócio-demográficas dos } \\
\text { utilizadores do e-mail }\end{array}$} \\
\hline \multirow[t]{2}{*}{ Características dos utilizadores } & \multicolumn{2}{|c|}{ Total } \\
\hline & $\mathrm{n}=92$ & $\%$ \\
\hline Sexo feminino & 67 & 72,8 \\
\hline \multicolumn{3}{|l|}{ Escolaridade } \\
\hline$>12$ anos & 60 & 65,2 \\
\hline Entre 8 e 12 anos & 27 & 29,3 \\
\hline Estudantes secundário & 3 & 3,3 \\
\hline \multicolumn{3}{|l|}{ Actividade } \\
\hline Activos & 76 & 82,6 \\
\hline Estudantes & 8 & 8,7 \\
\hline Reformados & 6 & 6,5 \\
\hline
\end{tabular}

As 504 mensagens foram enviadas por 92 utilizadores diferentes, o que corresponde uma média 5,5 mensagens por utilizador (mínimo de 1, máximo de 40, mediana de 3 e desvio-padrão de 6,6). Todos os utilizadores tinham previamente consultado a MF no CS. A sua média de idade era de 37,1 anos (mínimo de 13 e máximo de 67, mediana de 35 com desvio-padrão de 11,5), $73 \%$ eram do sexo feminino e $65 \%$ tinham escolaridade de nível superior (Quadro I). Os 92 utilizadores pertenciam a 79 agregados familiares diferentes, $73,4 \%$ dos quais de tipo nuclear.

No mesmo período, foram enviadas pela MF 462 mensagens, cinco das quais colectivas (a todos os pacientes da lista de contactos).

\section{Caracterização do conteúdo das mensagens}

As 100 mensagens da amostra aleatória das mensagens recebidas foram escritas acerca de 112 sujeitos (média de 1,1 sujeitos por mensagem, mínimo de uma e máximo de quatro), registando-se que estas diziam respeito, por ordem de frequência, ao próprio $(n=61)$, um filho menor ( $n=23)$, um idoso em situação de dependência $(n=12)$, ao cônjuge $(n=11)$ e a outros familiares não dependentes $(n=5)$.

O número de palavras por mensagem foi em média 57,1 (mínimo de 1, máximo de 483, mediana de 40 com desvio-padrão de 65,9). Apenas duas mensagens tinham mais de 300 palavras e 1 entre 150 e 300 . As 100 mensagens abordavam um total de 130 assuntos (média de 1,3 assuntos por mensagem), sendo que em 73
QUADRO II. Assuntos abordados nas mensagens

recebidas e respectiva frequência

\begin{tabular}{l|c|c} 
Assuntos abordados & \multicolumn{2}{|c}{ Total de mensagens } \\
\hline Questões médicas & $\mathbf{n = 9 2}$ & $\mathbf{\%}$ \\
\hline Medicação & 20 & 15,4 \\
\hline Exames complementares & 19 & 14,6 \\
\hline Administrativo/enfermagem & 19 & 14,6 \\
\hline Actualização sobre problema & 19 & 14,6 \\
\hline Actualização sobre referenciação & 16 & 12,3 \\
\hline Atestados & 7 & 5,4 \\
\hline Outros & 6 & 4,6 \\
\hline Total & 24 & 18,5 \\
\hline
\end{tabular}

mensagens era abordado apenas um assunto, em 24 mensagens eram abordados dois assuntos e apenas três mensagens abordavam três assuntos cada. Os assuntos mais frequentemente abordados foram as questões médicas $(n=20)$, os relacionados com exames complementares de diagnóstico $(\mathrm{n}=19)$, as questões administrativas e de enfermagem $(n=19)$, os relacionados com a medicação $(n=19)$ e as actualizações relativas a problemas já conhecidos ( $\mathrm{n}=16)$ (Quadro II).

A MF respondeu a 69 das 100 mensagens recebidas da amostra. As 31 mensagens não respondidas continham 35 assuntos, a maioria dos quais $(77,2 \%)$ eram exclusivamente de agradecimento ou assentimento ou de actualização. A maioria das mensagens (52,2\%) foi respondida entre 12 e 48 horas após a sua recepção, sendo $42 \%$ respondidas num período até 12 horas e apenas 5,8\% respondidas após 48 horas. O número médio de palavras das mensagens enviadas foi de 58,2 (mínimo de uma e máximo de 505, mediana 38 e desviopadrão 73,7), com cinco mensagens entre 150 e 300 palavras e apenas uma com mais de 300.

Cerca de $1 / 5$ das mensagens recebidas (22\%) foi escrito pelos utilizadores fora do horário da abertura do CS, sendo $37 \%$ escritas durante o horário de consulta da MF. Quarenta e dois por cento das respostas da MF foram enviadas dentro do seu horário de consulta, sendo os restantes (58\%) enviados no seu horário de trabalho não assistencial.

Quase um 1/3 das mensagens ( $n=29)$ não resultaram em qualquer acção para a MF. As restantes 71 men- 
QUADRO III. Actos médicos resultantes das mensagens recebidas

Actos médicos Total de mensagens

\begin{tabular}{l|c|c}
\hline & $\mathbf{n = 9 2}$ & $\%$ \\
\hline Aconselhamento/informação médica & 35 & 38,9 \\
\hline Receita /credencial MFR* & 18 & 20 \\
\hline Marcação consulta & 16 & 17,8 \\
\hline Exames complementares diagnóstico & 7 & 7,8 \\
\hline Referenciação hospitalar & 5 & 5,5 \\
\hline Outros & 9 & 9,9 \\
\hline & 90 & 99,9
\end{tabular}

*Medicina Física e Reabilitação

sagens resultaram em 90 acções pela MF (1,3 resultados por mensagem, máximo de quatro): resposta com troca de informação médica e/ou aconselhamento $(n=35)$, emissão de receitas, credenciais para MCD e atestados $(n=28)$, marcação de consulta $(n=16)$, referenciação hospitalar $(n=5)$ e outros $(n=6)$ (Quadro III).

\section{Avaliação do tempo gasto com o e-mail}

Na semana de 16 a 20 de Junho, em cinco dias de trabalho efectivo, a MF gastou um total de 27 minutos e 36 segundos a ler 18 mensagens e a enviar 16 em resposta, a que corresponde um dispêndio diário cinco minutos e 31 segundos (mínimo 12 segundos e máximo de 11 minutos e 25 segundos) para uma média de 3,6 mensagens recebidas e 3,2 mensagens enviadas diariamente (ou de 92 segundos por cada mensagem recebida e envio da respectiva resposta, quando necessário).

\section{Contextualização da prática clínica}

O CS onde decorreu o estudo situa-se na Senhora da Hora (localidade recentemente elevada a cidade), no concelho de Matosinhos, confinando a sul com os limites da cidade do Porto. A área de influência do CS compreende três freguesias: duas de características suburbanas (Senhora da Hora e Custóias) e uma de características rurais (Guifões). De entre os cerca de 56.000 inscritos no CS, a 19/11/2008 pertenciam à lista da MF autora 1.586 utentes, 130 dos quais (8.2\%) tinham 65 ou mais anos. Trata-se de uma lista pouco envelhecida, se comparada com a população inscrita no CS (em 2007, $13.4 \%$ dos inscritos tinham 65 ou mais anos). A lista tem uma taxa de analfabetismo de $3,7 \%$ (inferior à do concelho de Matosinhos), tendo $26,5 \%$ dos utentes escolaridade superior a 12 anos. A taxa de utilização dos utentes da lista no ano de 2007 foi de $70 \%$.

Em Novembro de 2008 num estudo de consulta com a duração de uma semana, foram realizadas pela MF um total de 84 consultas presenciais, 20 das quais a pacientes não incluídos na sua lista (consulta de recurso). No mesmo período, foram estabelecidos 64 contactos não presenciais (12 dos quais, por inter-substituição, de pacientes não incluídos na sua lista). Dos 52 contactos de utentes da lista, 16 foram telefonemas, 12 foram $e$-mails, 20 foram ao balcão administrativo e quatro através de familiares consultados. A média diária foi de 30 contactos por dia (23 se considerados apenas os pacientes da lista), dos quais 17 foram presenciais (13 se considerada apenas a lista de utentes).

\section{DISCUSSÃO}

Neste estudo de caracterização do uso de $e$-mail entre uma MF e os seus pacientes, verificou-se uma adesão crescente de utilizadores ao longo de 14 meses. Foram sobretudo as mulheres e os mais escolarizados os que enviaram mensagens, quase sempre curtas e sobre um único assunto médico, relativo a si próprio/a ou a um dependente, mensagens às quais a MF respondeu rapidamente e sem significativa sobrecarga de trabalho.

Como pontos fortes do presente estudo salientam-se: ainda não terem sido publicados em Portugal estudos sobre o uso do $e$-mail entre médicos e pacientes; ter sido estudado o movimento total de mensagens ao longo de mais de um ano e as características sócio-demográficas de todos os utilizadores; a fonte de grande parte dos dados ser electrónica; e a colheita dos dados ser da responsabilidade de um só investigador eliminando o viés inter-observador.

Como possíveis vieses, devem considerar-se os seguintes: a colheita dos dados subjectivos (como o assunto ou o resultado das mensagens) ter sido feita pela MF que recebeu e enviou as mensagens, o que poderá, pelo conhecimento que tem das situações clínicas em causa, introduzir um viés de observador; a inexistência de critérios precisos para classificação de duas variáveis qualitativas (dos assuntos e dos resultados das mensagens) poderá introduzir também um viés de classificação. 
Foram encontrados muitos estudos realizados nos Estados Unidos acerca do uso do $e$-mail entre médicos e pacientes (alguns envolvendo MF e também pediatras e internistas comunitários, ${ }^{9,13,15,17,18,24,26}$ outros não especificando a especialidade médica ${ }^{11,12,16} \mathrm{e}$ um referente a múltiplas especialidades e contextos de trabalho).${ }^{14} \mathrm{Foi}$ encontrada apenas uma referência a um estudo europeu (escocês), embora apenas divulgado numa carta ao director. ${ }^{10}$ De entre os referidos, alguns são estudos de metodologia semelhante à presente (avaliação objectiva da troca de mensagens), outros basearam-se em inquéritos a pacientes e/ou a profissionais de saúde, outros ainda misturam ambas as metodologias.

A adesão dos pacientes da lista ao $e$-mail para contacto com a MF cifrou-se, ao fim de 14 meses, em 5,8\% dos pacientes da lista. Este valor já suplanta o referido num estudo que revelou que a maioria dos médicos só usa o correio electrónico com 1 a $5 \%$ dos seus pacientes, ${ }^{22}$ prevendo-se mesmo que, caso se mantenha a tendência crescente, venha a atingir os $9,2 \%$ obtidos no estudo de Liederman. ${ }^{13}$

De encontro ao verificado noutros estudos, as mulheres são mais utilizadoras ${ }^{12,13,15} \mathrm{e}$, tipicamente, têm um grau de escolaridade intermédio a superior. ${ }^{9,12,13,15} \mathrm{~A}$ dimensão média das mensagens recebidas foi neste estudo semelhante à das enviadas, enquanto que, no único estudo disponível que analisou esta variável, a dimensão das mensagens recebidas foi mais do dobro (139 vs 57 palavras e $10 \%$ vs $2 \%$ com mais de 300 ) e a das enviadas foi quase metade (39 vs 58 palavras). ${ }^{26}$

Como encontrado noutros estudos, as mensagens abordam quase sempre um único assunto, mais frequentemente questões médicas, de medicação ou sobre MCD. ${ }^{9,12,13,15-18,25,26}$ Neste estudo, as mensagens recebidas resultaram em actos médicos em $2 / 3$ dos casos, sendo o mais frequente o fornecimento de informação ou aconselhamento e a emissão de receitas, credenciais de MCD e de atestados. No único estudo disponível que avaliou a esta variável, $42 \%$ das trocas de mensagens resultaram em prescrições, $26 \%$ em telefonemas, $11 \%$ em consultas e $5 \%$ em referenciações (estas diferenças podem explicar-se pelo facto de o estudo referido ser sobre o uso do $e$-mail entre pediatras e pais de crianças).${ }^{15} \mathrm{~A}$ rapidez das mensagens de resposta da MF ( $42 \%$ em 12 horas e $94,2 \%$ em 48 horas) foi uma constante também noutros estudos: $83 \%$ até às 48 horas no estudo de Leong, ${ }^{9} 85 \%$ em 16 horas (de expediente) ou menos no estudo de Liederman e Lee, ${ }^{13}$ e $59 \%$ em menos de 24 horas (e média de 31 horas) no de Sittig. ${ }^{26}$ É de referir que a existência de uma resposta e a respectiva rapidez foram factores determinantes na satisfação dos pacientes utilizadores de $e$-mail nos estudos de Houston e de Liederman. ${ }^{12,13,17}$

$\mathrm{O}$ número médio de mensagens recebidas diariamente $(2,08)$ foi semelhante ao encontrado em alguns estudos $^{9,15,26}$ (2,2 no estudo de Leong e 2,6 no de Sittig $2,6)$ mas superior ao encontrado $(1,2)$ nos dois anos que durou o estudo de Rosen sobre o uso de $e$-mail entre pediatras e pais de crianças $^{18} \mathrm{e}$ às 10 mensagens mensais estimadas pelos médicos do estudo de Gaster. ${ }^{14}$ O tempo dispendido pela MF (em média 92 segundos por mensagem recebida) diferiu pouco do encontrado em outros estudos: um a cinco minutos/mensagem no estudo de Leong; ${ }^{9}$ e uma média de dois minutos por mensagem $v s$ cinco minutos por telefonema gastos pelos pediatras do estudo de Rosen. ${ }^{18}$ Tal como noutros estudos, os pacientes aproveitam horários para além do assistencial para contactarem o seu MF por $e$-mail..$^{15,18}$

Tratando-se de uma forma de acesso aos cuidados de saúde que ainda não está generalizada na MGF portuguesa, é importante salientar que estes resultados parecem não dar razão ao medo de sobre-utilização dos pacientes e de sobrecarga de trabalho que motivam a não adopção do sistema por alguns médicos. Simultaneamente, podem fazer luz sobre o desconhecido que poderá impedir outros de aderir.

Seria interessante verificar, através da realização de mais estudos semelhantes, se os resultados se reproduziriam em populações de pacientes e de MF de características diversas. Complementarmente, seria interessante comparar a satisfação dos pacientes e dos MF que podem comunicar entre si por $e$-mail com a daqueles que não têm essa possibilidade.

À luz do que tem sido feito em outros países, seria ainda importante, para a generalização desta forma de comunicação a elaboração - por entidade competente - de normas de orientação clínica (para salvaguardar questões clínicas, éticas e médico-legais); ${ }^{26-28}$ a integração da funcionalidade de $e$-mail no processo clínico electrónico dos pacientes; ${ }^{23} \mathrm{e}$, decorrente da anterior, a contabilização dos contactos por $e$-mail para efeitos de produtividade. . $^{323,30}$ 


\section{REFERÊNCIAS BIBLIOGRÁFICAS}

1. European Academy of Teachers in General Practice. A Definição Europeia de Medicina Geral e Familiar. Rev Port Clin Geral 2005; 21: 511-6.

2. Sá AB. Filosofia e prática da Clínica Geral: consequências da sua especificidade. Rev Port Clin Geral 1995;12: 47-53.

3. Gérvas J, Bonis J. El debate profesional acerca de la escasez de médicos. Rev Esp Salud Pública 2008 Nov-Dec; 82 (6): 627-35.

4. Haggerty JL, Pineault R, Beaulieu MD, Brunelle Y, Gauthier J, Goulet F, et al. Practice features associated with patient-reported accessibility, continuity, and coordination of primary health care. Ann Fam Med 2008 Mar-Apr; 6 (2): 116-23.

5. Baleiras $\mathrm{SJ}$, Ramos V. A gestão da prática clínica pelo médico de família. Rev Port Clin Ger 1992; 9 (4): 116-24.

6. Fortin M, Maltais D, Hudon C, Lapointe L, Ntetu AL. Access to health care: perceptions of patients with multiple chronic conditions. Can Fam Physician 2005 Nov; 51: 1502-3.

7. Cerejo AC, Ponte C, Marques I, Santos R, Trancoso J, Sanjurjo A, Et al. Satisfação dos utentes do Centro de Saúde da Senhora da Hora. Comunicação livre ( $1^{\circ}$ prémio categoria investigação) no $11^{\circ}$ Congresso Nacional de Medicina Familiar; 2006 Setembro, 24-26; Aveiro, Portugal.

8. Committee on Quality Health Care, Institute of Medicine. Crossing the Quality Chasm: a new health system for the 21st century. Washington, DC: National Academy Press; 2001. Disponível em: http://books.nap.edu/html/quality_chasm/reportbrief.pdf [acedido em 24/09/2009].

9. Leong SL, Gingrich D, Lewis PR, Mauger DT, George JH. Enhancing doctor-patient communication using email: a pilot study. J Am Board Fam Pract 2005 May-Jun; 18 (3): 180-8.

10. Neville R, Marsden W, McCowan C, Pagliari C, Mullen H, Fannin A. E-mail consultations in general practice. Br J Gen Pract 2004 Jul; 54 (504): 546.

11. Hereford J, Bell E, Lee J, Eytan T. Patient satisfaction with a web-based patient-provider messaging system implemented across a statewide delivery system (Group Health Cooperative). AMIA Annu Symp Proc 2003: 860.

12. Houston TK, Sands DZ, Jenckes MW, Ford DE. Experiences of patients who were early adopters of electronic communication with their physician: satisfaction, benefits, and concerns. Am J Manag Care 2004 Sep; 10 (9): 601-8.

13. Liederman EM, Lee JC, Baquero VH, Seites PG. Patient-physician web messaging: the impact on message volume and satisfaction. J Gen Intern Med 2005 Jun; 20 (1): 52-7.

14. Gaster B, Knight CL, DeWitt DE, Sheffield JV, Assefi NP, Buchwald D. Physician's use and attitudes toward electronic mail for patient communication. J Gen Intern Med 2003 May; 18 (5): 385-9.

15. Anand SG, Feldman MJ, Geller DS, Bisbee A, Bauchner H. A content analysis of e-mail communication between primary care providers and parents. Pediatrics 2005 May; 115: 1283-8.

16. Patt MR, Houston TK, Jenckes MW, Sands DZ, Ford DE. Doctors who are using e-mail with their patients: a qualitative exploration. J Med Internet Res 2003 Apr-Jun; 5 (2): e9. doi: 10.2196/jmir.5.2.e9.

17. Liederman EM, Morefield CS. Web messaging: a new tool for patientphysician communication. J Am Med Inform Assoc 2003 May-Jun; 10 (3): 260-70.
18. Rosen P, Kwoh CK. Patient-physician e-mail: an opportunity to transform pediatric health care delivery. Pediatrics 2007 Oct; 120 (4):701$-6$.

19. Harris Interactive. Many patients willing to pay for online communication with their physicians [press release]. Rochester, NY: Harris Interactive; April 11, 2002. Disponível em: http://www.harrisinteractive.com/news/allnewsbydate.asp? NewsID $=446$ [acedido em 15/03/2009].

20. Delbanco T, Sands DZ. Electrons in flight: e-mail between doctors and patients. N Engl J Med 2004 Apr 22; 350 (17): 1705-7.

21. Eurostat. Households who have Internet access at home. 2008. Disponível em: http://epp.eurostat.ec.europa.eu/tgm/table.do?tab=table \&init $=1 \&$ language $=$ en $\& p c o d e=$ tin $00088 \&$ plugin $=0$ [acedido em 25/09/2009].

22. Pizziferri L, Kittler A, Volk LA, Hobbs J, Jagannath Y, Wald JS, et al. Physician's perceptions towards electronic communication with patients. AMIA Annu Symp Proc 2003: 972.

23. Stone JH. Communication between physicians and patients in the era of E-medicine. N Engl J Med 2007 Jun 14; 356 (24): 2451-4.

24. Gerstle RS, American Academy of PediatriciAns Task Force on Medical Informatics. E-mail communication between pediatricians and their patients. Pediatrics 2004 Jul; 114 (1): 317-21.

25. White CB, Moyer CA, Stern DT, Katz SJ. A content analysis of e-mail communication between patients and their providers: patients get the message. J Am Med Inform Assoc 2004 Jul-Aug; 11 (4): 260-7.

26. Sittig DF. Results of a content analysis of electronic messages (e-mail) sent between patients and their physicians. BMC Med Inform Decis Mak 2003 Oct 1; 3: 11.

27. Sands K. Guidelines for the clinical use of electronic mail with patients. J Am Med Inform Assoc 1998 Jan-feb; 5 (1): 104-11.

28. Physician Guidelines for Online Communication with Patients. Canadian Medical Association; 2005.

29. American Medical Association. Guidelines for Physician-Patient Electronic Communications. Disponível em http://www.ama-assn.org/ ama/pub/category/2386.html [acedido em 09/062008].

30. Eads M. Virtual office visits: a reachable and reimbursable innovation. Fam Pract Manag 2007 Oct; 14 (9): 20-2.

A autora declarou não possuir conflitos de interesses

\section{ENDEREÇO PARA CORRESPONDÊNCIA}

Mónica Granja

Rua da Lagoa, sem $\mathrm{n}^{\circ}$

4460-352 Sra. da Hora

E-mail: monica.granja@ulsm.min-saude.pt

Recebido em 29/09/2009

Aceite para publicação em 08/12/2009 


\section{ABSTRACT}

\section{E-MAIL USE BETWEEN A FAMILY PHYSICIAN AND HER PATIENTS: A FOURTEEN MONTH EXPERIENCE}

Objectives: To evaluate message exchange, between a family physician and her patients, during the first fourteen months of e-mail access.

Study Design: Observational cross-sectional.

Setting: A family practice in Senhora da Hora, Matosinhos, Portugal.

Population: E-mail messages exchanged between physician and patients.

Methods: A random sample of $20 \%$ of patient messages was evaluated for: person the message was written about, size, number and type of subjects mentioned, whether or not it elicited a reply from the family physician and, if so, the reply message size and time between initial message and reply, time of day messages were sent and message results. Socio-demographic and familiar data were collected for every patient using e-mail. The amount of time spent by family physician adressing e-mail during a whole week was measured. For descriptive statistical analysis the author used Excel 2003.

Results: A total of 504 messages were received from 92 different patients, whose mean age was 37.1, being 73\% of which female and 65\% with higher education. The family physician herself sent 462 messages. Messages studied ( $\mathrm{n}=100)$ from patients were written about 112 persons (most often the one who wrote the message, children, dependent elderly or spouse). Mean message size was 57.1 words and mentioned a mean of 1.3 subjects (most often medical questions, test results, nursing or administrative procedures and medication). The family physician replied to $69 \%$ of the messages received, doing so in less than 48 hours for $94.2 \%$ of cases and using a mean of 58.2 words per message. Messages from patients were written out of hours in $22 \%$ of cases. The family physician sent $58 \%$ of replies during non-consultation time. Most often, the messages results were: reply with medical information or advice, prescription fills, tests or treatment forms, certificates, appointment scheduling and referral. During a week, the family physician took an average of 5 minutes and 31 seconds daily to address e-mail. Conclusions: As in other studies conducted in the United States, this study found that patients and carers, mainly professionally active and higher educated female, adhere to e-mail to communicate with their family physician, without causing physician overload. These results may favour higher adherence of family physicians to e-mail and of family practices to invest in this tool.

Keywords: E-mail; Communication; Accessibility. 